\title{
Unpatterned Wafer Haze as a Monitor of Film Thickness Profile and Composition for Blanket Wafers Deposited by Atomic Layer Deposition
}

\author{
K. Beckwitt*, B. Donehoo*, T.B. Tran*, N.M. Perez-paz*, T.E. Glassman*, and C.D. Thomas* \\ * Portland Technology Development, Intel Corp., Hillsboro, OR 97124.
}

Laser scattering surface maps ('haze') collected from high volume unpatterned wafer inspection systems are gaining significance as a new tool to measure the wafer surface quality of bare and blanket film wafers used in semiconductor fabs. Historically, dark field microscopy was used to measure defect levels on unpatterned wafers by identifying isolated light scattering events, but the background scattering signal collected during inspection was treated primarily as a measure of wafer noise and was not monitored. Recent advancements in optical detection and signal processing have enabled the active collection and analysis of these haze data in real time during wafer defect inspection and numerous applications have been found to monitor substrate surface morphology [1], the quality of deposited and grown films [2], and other wafer processing techniques [3].

In this study, we demonstrate the applicability of unpatterned wafer haze as collected by commercially available UV unpatterned wafer inspection systems to monitor the thickness and composition of advanced chemical vapor deposition films used in Intel's manufacturing process at design rules of $32 \mathrm{~nm}$ and below.

Fig. 1(a) shows the haze response as a function of wafer mean film thickness for wafers deposited with 10's of angstroms of a front-end gate stack material from an advanced chemical vapor deposition tool. For this material stack, haze strongly correlates with film thickness and provides an excellent real-time monitor of deposition performance. In addition, for higher haze (and thicker) film thickness regions, haze indicates increased wafer noise and provides an inline diagnostic of measured defect signal quality as can be seen by the false defect regions at the highest haze levels in Fig. 1(b). Fig. 2 shows measured haze signal versus several thickness steps and reduction in $\%$, a key film component. Within each thickness level, mean wafer haze correlates to film composition.

The sensitivity of haze to film properties provides valuable diagnostics of process tool chamber performance as the deposited film properties change over chamber life and between cycles of preventative-maintenance. Here, trending haze over chamber life revealed marginality in the film deposition, enabling a process optimization that immediately improved the robustness of the film to the underlying issue and eliminated its haze and defect signature.

The capability of haze data to differentiate between isolated particles/defects and process induced roughness that manifests as localized defects is extremely beneficial during semiconductor process development. And in high-volume manufacturing environments, these data are a valuable metric for monitoring film quality since they are collected simultaneously with defect data and do not require additional measurement time or monitor wafers.

References

[1] D.K. Chen et al., IEEE International Symposium on Semiconductor Manufacturing (ISSM), 2527 Sept. 2006, 134-137.

[2] B. Arrazat et al., Microelectronic Engineering, 84 (2007) 2558-2562. 
[3] W.-Y. Teng et al., Advanced Semiconductor Manufacturing Conference, 11-12 June 2007, 6168.
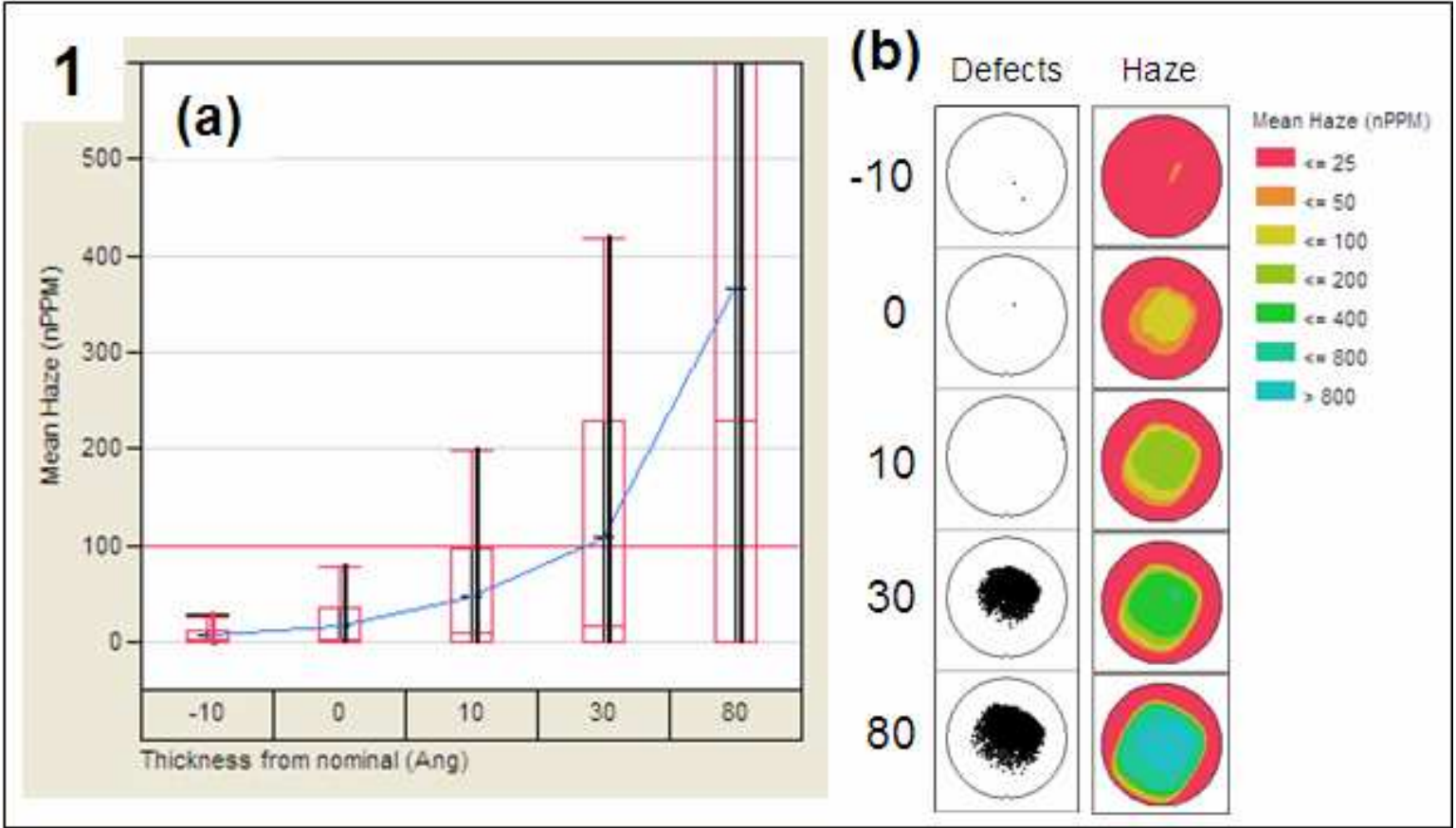

FIG. 1. Wafer haze distribution in normalized parts-per-million scatter by (a) offset in deposited film thickness from nominal and (b) spatial haze variation showing correlation between localized wafer haze and detected defect 'noise' at higher haze (higher thickness) values. In (a), the blue line indicates the wafer-mean haze for each thickness level and box-plots indicate variability. The red line at 100 nPPM scattering shows the upper range of linear scattering response.

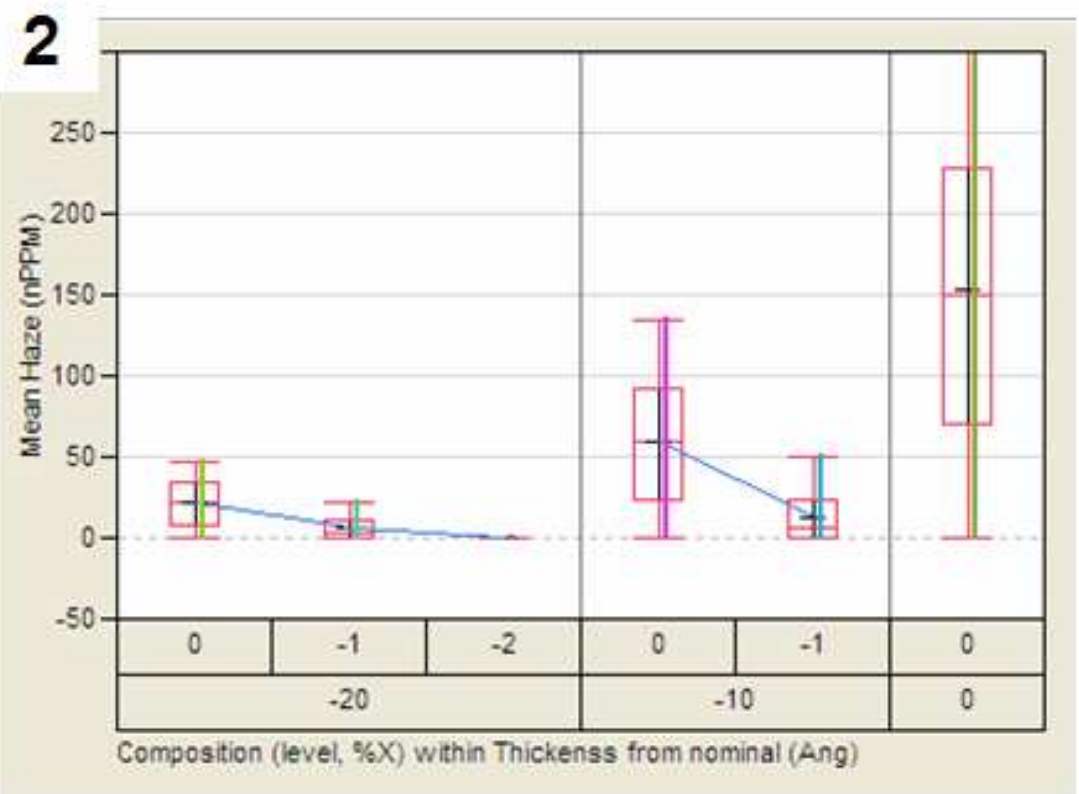

FIG. 2. Wafer haze distribution (nPPM) by film thickness (offset from nominal film thickness) and variation in composition of a key film component $(\mathrm{X})$. \% X levels are indicated by $0,-1,-2$ where 0 is nominal and $-1,-2$ are 2 levels of reduced $\% \mathrm{X}$. 